\title{
About Supergraphs. Part III
}

\author{
Sebastian Koch \\ Johannes Gutenberg University \\ Mainz, Germany ${ }^{1}$
}

\begin{abstract}
Summary. The previous articles [5] and 6] introduced formalizations of the step-by-step operations we use to construct finite graphs by hand. That implicitly showed that any finite graph can be constructed from the trivial edgeless graph $K_{1}$ by applying a finite sequence of these basic operations. In this article that claim is proven explicitly with Mizar [4.
\end{abstract}

MSC: 05C76 68T99 03B35

Keywords: supergraph; graph operations; construction of finite graphs MML identifier: GLIB_008, version: 8.1.09 5.57.1355

\section{INTRODUCTION}

In the literature a mutual understanding how the graphical representation of graphs is to be translated into a description fitting the set-theoretic definition is usually assumed (cf. [9], [3, [8], 2]), but in Mizar we need explicit operations, which were provided in [5] and [6].

The rather extensive preliminaries contain many theorems that would fit well into earlier articles of the GLIB series, for example:

- In a simple graph, the degree of a vertex equals the cardinality of its neighbors.

- The operations of removing a vertex or an edge in a graph commute.

- Every finite connected graph has a spanning tree.

\footnotetext{
${ }^{1}$ The author is enrolled in the Johannes Gutenberg University in Mayence, Germany, mailto: skoch02@students.uni-mainz.de 
- Endvertices are no cut vertices.

Graphs without edges are rigorously introduced in the following section. Wilson calls those null graphs ([9]). Bondy and Murty call them empty graphs ([3]), while naming the graph without vertices the null graph. Both notations are common in the literature. To avoid confusion those graphs are simply introduced as edgeless here.

To describe the construction of finite graphs starting from the trivial edgeless $K_{1}$, finite sequences yielding graphs are needed, which are introduced in the next section expanding the notation from [7, [1].

The last section contains the formalizations of the main results:

- Adding $n$ vertices to a graph can be done by adding one vertex after another.

- Any finite edgeless graph can be constructed from $K_{1}$ by adding one vertex at a time.

- Any finite (connected) graph can be reconstructed from a spanning (connected) subgraph by adding one edge at a time.

- Any finite graph can be constructed from $K_{1}$ by adding one vertex or one edge at a time.

- Any finite tree can be constructed from $K_{1}$ by adding one vertex and an edge incident with that vertex at a time.

- Any finite connected graph can be constructed from $K_{1}$ by adding one edge or one vertex and an edge incident with that vertex at a time.

- Adding a vertex to a graph and connecting it to a (possibly empty) subset of the vertices of said graph can be done by first adding the new vertex and then adding one edge at a time.

- Any finite simple graph can be constructed from $K_{1}$ by adding one vertex connecting it to a (possibly empty) subset of the vertices of the previous contruction step at a time.

- If the finite simple graph is also connected, the subset of adjacent vertices can be guarantied to be non empty.

The number of operations needed is given for each process in terms of order and size of the involved graphs. Some proof schemes are presented to make use of these constructions. 


\section{Preliminaries}

Let $G$ be a graph and $v$ be a vertex of $G$. Let us observe that every subgraph of $G$ induced by $\{v\}$ is trivial.

Let us consider a graph $G$, a set $X$, and a vertex $v$ of $G$. Now we state the propositions:

(1) $G$.edgesBetween $(X \backslash\{v\})=G$.edgesBetween $(X) \backslash v$.edgesInOut () .

(2) If $v$ is isolated, then $G$.edgesBetween $(X \backslash\{v\})=G$.edgesBetween $(X)$. The theorem is a consequence of (1).

Let us consider a non-directed-multi graph $G$ and a vertex $v$ of $G$. Now we state the propositions:

(3) $\quad v \cdot$ inDegree ()$=\overline{\overline{v \cdot \operatorname{inN} N e i g h b o r s()}}$.

Proof: Define $\mathcal{P}$ [object, object $] \equiv \$_{2}$ joins $\$_{1}$ to $v$ in $G$. Consider $f$ being a function such that $\operatorname{dom} f=v$.inNeighbors () and for every object $x$ such that $x \in v$.inNeighbors () holds $\mathcal{P}[x, f(x)] . f$ is a bijection between $v$.inNeighbors () and $v$.edgesIn( () .

(4) $v$.outDegree ()$=\overline{\overline{v \text {.outNeighbors }()}}$.

Proof: Define $\mathcal{P}$ [object, object $] \equiv \$_{2}$ joins $v$ to $\$_{1}$ in $G$. Consider $f$ being a function such that $\operatorname{dom} f=v$.outNeighbors() and for every object $x$ such that $x \in v$.outNeighbors () holds $\mathcal{P}[x, f(x)]$. $f$ is a bijection between $v$.outNeighbors() and $v$.edgesOut().

(5) Let us consider a simple graph $G$, and a vertex $v$ of $G$. Then $v$.degree ()$=$ $\overline{v \text {.allNeighbors }()}$.

PRoOF: $v \cdot \operatorname{inNeighbors}() \cap v$.outNeighbors ()$=\emptyset$.

(6) Let us consider a graph $G$. Then $G$ is loopless if and only if for every vertex $v$ of $G, v \notin v$.allNeighbors () .

Proof: For every object $v$, there exists no object $e$ such that $e$ joins $v$ and $v$ in $G$.

(7) Let us consider a graph $G$, and a vertex $v$ of $G$. Then $v$ is isolated if and only if $v$.allNeighbors ()$=\emptyset$.

(8) Let us consider a graph $G_{1}$, a set $v$, and a subgraph $G_{2}$ of $G_{1}$ with vertex $v$ removed. Suppose $G_{1}$ is trivial or $v \notin$ the vertices of $G_{1}$. Then $G_{1} \approx G_{2}$.

(9) Let us consider graphs $G_{1}, G_{2}$, and a set $v$. Suppose $G_{1} \approx G_{2}$ and ( $G_{1}$ is trivial or $v \notin$ the vertices of $\left.G_{1}\right)$. Then $G_{2}$ is a subgraph of $G_{1}$ with vertex $v$ removed.

(10) Let us consider a graph $G$. Suppose there exist vertices $v_{1}, v_{2}$ of $G$ such that $v_{1} \neq v_{2}$. Then $G$ is not trivial.

Proof: $\overline{\bar{\alpha}} \neq 1$, where $\alpha$ is the vertices of $G$. 
Let $G$ be a non trivial graph and $X$ be a set. Let us note that every subgraph of $G$ with edges $X$ removed is non trivial. Now we state the propositions:

(11) Let us consider a finite graph $G_{1}$, and a subgraph $G_{2}$ of $G_{1}$. Then $G_{2}$ is spanning if and only if $G_{1}$.order ()$=G_{2}$.order () .

(12) Let us consider a graph $G_{1}$, and a spanning subgraph $G_{2}$ of $G_{1}$. Suppose the edges of $G_{1}=$ the edges of $G_{2}$. Then $G_{1} \approx G_{2}$.

(13) Let us consider a finite graph $G_{1}$, and a spanning subgraph $G_{2}$ of $G_{1}$. If $G_{1} \cdot \operatorname{size}()=G_{2} \cdot \operatorname{size}()$, then $G_{1} \approx G_{2}$. The theorem is a consequence of (12).

(14) Let us consider a graph $G_{1}$, a set $V$, and a subgraph $G_{2}$ of $G_{1}$ induced by $V$. If $G_{2}$ is spanning, then $G_{1} \approx G_{2}$.

Let us consider a graph $G$. Now we state the propositions:

(15) $G$ is not trivial if and only if there exists a subgraph $H$ of $G$ such that $H$ is not spanning.

(16) If there exists a vertex $v$ of $G$ such that $v$ is endvertex, then $G$ is not trivial.

Proof: Consider $e$ being an object such that $v$.edgesInOut ()$=\{e\}$ and $e$ does not join $v$ and $v$ in $G$. For every vertex $u$ of $G$, the vertices of $G \neq\{u\}$.

(17) Let us consider a graph $G_{1}$, sets $v, e$, a subgraph $G_{2}$ of $G_{1}$ with vertex $v$ removed, and a subgraph $G_{3}$ of $G_{1}$ with edge $e$ removed. Then every subgraph of $G_{2}$ with edge $e$ removed is a subgraph of $G_{3}$ with vertex $v$ removed. The theorem is a consequence of (1), (8), and (9).

(18) Let us consider a graph $G_{1}$, sets $v$, e, a subgraph $G_{2}$ of $G_{1}$ with edge $e$ removed, and a subgraph $G_{3}$ of $G_{1}$ with vertex $v$ removed. Then every subgraph of $G_{2}$ with vertex $v$ removed is a subgraph of $G_{3}$ with edge $e$ removed. The theorem is a consequence of (1) and (8).

Let $G$ be a finite, connected graph. Note that there exists a subgraph of $G$ which is spanning, tree-like, connected, and acyclic.

Now we state the propositions:

(19) Let us consider a connected graph $G_{1}$, and a subgraph $G_{2}$ of $G_{1}$. Suppose the edges of $G_{1} \subseteq$ the edges of $G_{2}$. Then $G_{1} \approx G_{2}$.

Proof: The vertices of $G_{1}=$ the vertices of $G_{2}$.

(20) Let us consider a finite, connected graph $G_{1}$, and a subgraph $G_{2}$ of $G_{1}$. If $G_{1} \cdot \operatorname{size}()=G_{2} \cdot \operatorname{size}()$, then $G_{1} \approx G_{2}$. The theorem is a consequence of (19).

(21) Let us consider a finite, tree-like graph $G_{1}$, and a spanning, tree-like subgraph $G_{2}$ of $G_{1}$. Then $G_{1} \approx G_{2}$. The theorem is a consequence of (11) 
and (13).

Let $G$ be a non trivial graph. Observe that there exists a subgraph of $G$ which is non spanning, trivial, and connected.

Now we state the propositions:

(22) Let us consider a graph $G$, and vertices $v_{1}, v_{2}$ of $G$. Suppose $v_{1} \notin$ G.reachableFrom $\left(v_{2}\right)$.

Then G.reachableFrom $\left(v_{1}\right)$ misses G.reachableFrom $\left(v_{2}\right)$.

(23) Let us consider a graph $G$. Then $G$.componentSet() is a partition of the vertices of $G$.

Proof: Set $V=$ the vertices of $G$. For every subset $A$ of $V$ such that $A \in G$.componentSet() holds $A \neq \emptyset$ and for every subset $B$ of $V$ such that $B \in G$.componentSet() holds $A=B$ or $A$ misses $B$.

(24) Let us consider a graph $G$, a partition $C$ of the vertices of $G$, and a vertex $v$ of $G$. If $C=G$.componentSet(),

then $\operatorname{EqClass}(v, C)=G \cdot \operatorname{reachableFrom}(v)$.

(25) Let us consider a graph $G_{1}$, vertices $v_{0}, v_{1}$ of $G_{1}$, a subgraph $G_{2}$ of $G_{1}$ with vertex $v_{0}$ removed, and a vertex $v_{2}$ of $G_{2}$. Suppose $v_{0}$ is endvertex and $v_{1}=v_{2}$ and $v_{1} \in G_{1}$.reachableFrom $\left(v_{0}\right)$. Then $G_{2}$.reachableFrom $\left(v_{2}\right)=$ $\left(G_{1}\right.$.reachableFrom $\left.\left(v_{1}\right)\right) \backslash\left\{v_{0}\right\}$.

Proof: $G_{1}$ is not trivial. For every object $w, w \in G_{2}$.reachableFrom $\left(v_{2}\right)$ iff $w \in G_{1}$.reachableFrom $\left(v_{1}\right)$ and $w \notin\left\{v_{0}\right\}$.

(26) Let us consider a non trivial graph $G_{1}$, vertices $v_{0}, v_{1}$ of $G_{1}$, a subgraph $G_{2}$ of $G_{1}$ with vertex $v_{0}$ removed, and a vertex $v_{2}$ of $G_{2}$. Suppose $v_{1}=v_{2}$ and $v_{1} \notin G_{1}$.reachableFrom $\left(v_{0}\right)$. Then $G_{2}$.reachableFrom $\left(v_{2}\right)=$ $G_{1}$.reachableFrom $\left(v_{1}\right)$.

Proof: For every object $w$ such that $w \in G_{1}$.reachableFrom $\left(v_{1}\right)$ holds $w \in G_{2}$.reachableFrom $\left(v_{2}\right)$.

(27) Let us consider a non trivial, finite, tree-like graph $G$, and a vertex $v$ of $G$. If $G$.order ()$=2$, then $v$ is endvertex.

Let $G$ be a non trivial, connected graph and $v$ be a vertex of $G$. Observe that $v$.allNeighbors() is non empty.

Now we state the propositions:

(28) Let us consider a tree $T$, and a vertex $a$ of $T$. Then $T \cdot \operatorname{pathBetween}(a, a)=$ $T \cdot w a l k O f(a)$.

(29) Let us consider a tree $T$, vertices $a, b$ of $T$, and an object $e$. If $e$ joins $a$ and $b$ in $T$, then $T$.pathBetween $(a, b)=T$.walkOf $(a, e, b)$.

(30) Let us consider a non trivial, finite tree $T$, and a vertex $v$ of $T$. Then there exist vertices $v_{1}, v_{2}$ of $T$ such that 
(i) $v_{1} \neq v_{2}$, and

(ii) $v_{1}$ is endvertex, and

(iii) $v_{2}$ is endvertex, and

(iv) $v \in\left(T \cdot \operatorname{pathBetween}\left(v_{1}, v_{2}\right)\right)$.vertices () .

Proof: Define $\mathcal{P}$ [natural number $] \equiv$ for every non trivial, finite tree $T$ for every vertex $v$ of $T$ such that $T$.order ()$=\$_{1}+2$ there exist vertices $v_{1}, v_{2}$ of $T$ such that $v_{1} \neq v_{2}$ and $v_{1}$ is endvertex and $v_{2}$ is endvertex and $v \in\left(T \cdot \operatorname{pathBetween}\left(v_{1}, v_{2}\right)\right)$.vertices () . $\mathcal{P}[0]$. For every natural number $k$ such that $\mathcal{P}[k]$ holds $\mathcal{P}[k+1]$. For every natural number $k, \mathcal{P}[k]$. Consider $k$ being a natural number such that $T$.order ()$=2+k$.

(31) Let us consider a non trivial, finite, tree-like graph $G_{1}$, and a non spanning, connected subgraph $G_{2}$ of $G_{1}$. Then there exists a vertex $v$ of $G_{1}$ such that

(i) $v$ is endvertex, and

(ii) $v \notin$ the vertices of $G_{2}$.

The theorem is a consequence of (30).

(32) Let us consider graphs $G_{2}, G_{3}$, a set $V$, and a supergraph $G_{1}$ of $G_{2}$ extended by the vertices from $V$. Suppose $G_{2} \approx G_{3}$. Then $G_{1}$ is a supergraph of $G_{3}$ extended by the vertices from $V$.

(33) Let us consider a graph $G_{2}$, and a supergraph $G_{1}$ of $G_{2}$. Suppose the edges of $G_{1}=$ the edges of $G_{2}$. Then $G_{1}$ is a supergraph of $G_{2}$ extended by the vertices from (the vertices of $\left.G_{1}\right) \backslash\left(\right.$ the vertices of $G_{2}$ ).

(34) Let us consider a finite graph $G_{1}$, and a subgraph $G_{2}$ of $G_{1}$. Suppose $G_{1} \cdot \operatorname{size}()=G_{2} \cdot \operatorname{size}()$. Then $G_{1}$ is a supergraph of $G_{2}$ extended by the vertices from (the vertices of $\left.G_{1}\right) \backslash\left(\right.$ the vertices of $G_{2}$ ). The theorem is a consequence of (33).

(35) Let us consider a non trivial graph $G_{1}$, a vertex $v$ of $G_{1}$, and a subgraph $G_{2}$ of $G_{1}$ with vertex $v$ removed. If $v$ is isolated, then $G_{1}$ is a supergraph of $G_{2}$ extended by $v$. The theorem is a consequence of (2).

(36) Let us consider graphs $G_{2}, G_{3}$, objects $v_{1}, e, v_{2}$, and a supergraph $G_{1}$ of $G_{2}$ extended by $e$ between vertices $v_{1}$ and $v_{2}$. Suppose $G_{2} \approx G_{3}$. Then $G_{1}$ is a supergraph of $G_{3}$ extended by $e$ between vertices $v_{1}$ and $v_{2}$.

(37) Let us consider a graph $G_{1}$, a set $e$, and a subgraph $G_{2}$ of $G_{1}$ with edge $e$ removed. Suppose $e \in$ the edges of $G_{1}$. Then $G_{1}$ is a supergraph of $G_{2}$ extended by $e$ between vertices (the source of $\left.G_{1}\right)(e)$ and (the target of $\left.G_{1}\right)(e)$.

ProOF: Set $u=\left(\right.$ the source of $\left.G_{1}\right)(e)$. Set $w=\left(\right.$ the target of $\left.G_{1}\right)(e)$. For every object $e_{0}$ such that $e_{0} \in \operatorname{dom}\left(\right.$ the source of $G_{1}$ ) holds (the source of 
$\left.G_{1}\right)\left(e_{0}\right)=\left(\left(\right.\right.$ the source of $\left.\left.G_{2}\right)+\cdot(e \mapsto \cdot u)\right)\left(e_{0}\right)$. For every object $e_{0}$ such that $e_{0} \in \operatorname{dom}$ (the target of $\left.G_{1}\right)$ holds (the target of $\left.G_{1}\right)\left(e_{0}\right)=(($ the target of $\left.\left.G_{2}\right)+\cdot(e \longmapsto w)\right)\left(e_{0}\right)$.

(38) Let us consider a non trivial graph $G_{1}$, a vertex $v$ of $G_{1}$, an object $e$, and a subgraph $G_{2}$ of $G_{1}$ with vertex $v$ removed. Suppose $\{e\}=v$.edgesInOut() and $e$ does not join $v$ and $v$ in $G_{1}$. Then $G_{1}$ is supergraph of $G_{2}$ extended by $v \cdot \operatorname{adj}(e), v$ and $e$ between them or supergraph of $G_{2}$ extended by $v$, $v \operatorname{adj}(e)$ and $e$ between them. The theorem is a consequence of (1).

(39) Let us consider a graph $G_{2}$, vertices $v_{1}, v_{2}$ of $G_{2}$, an object $e$, a supergraph $G_{1}$ of $G_{2}$ extended by $e$ between vertices $v_{1}$ and $v_{2}$, a vertex $w$ of $G_{1}$, and a vertex $v$ of $G_{2}$. Suppose $v_{2} \in G_{2}$.reachableFrom $\left(v_{1}\right)$ and $v=w$. Then $G_{1} \cdot \operatorname{reachableFrom}(w)=G_{2}$.reachableFrom $(v)$.

(40) Let us consider a graph $G_{2}$, vertices $v_{1}, v_{2}$ of $G_{2}$, an object $e$, and a supergraph $G_{1}$ of $G_{2}$ extended by $e$ between vertices $v_{1}$ and $v_{2}$. Suppose $v_{2} \in$ $G_{2}$.reachableFrom $\left(v_{1}\right)$. Then $G_{1} \cdot \operatorname{componentSet}()=G_{2} \cdot \operatorname{componentSet}()$. The theorem is a consequence of (39).

(41) Let us consider a graph $G_{2}$, vertices $v_{1}, v_{2}$ of $G_{2}$, an object $e$, a supergraph $G_{1}$ of $G_{2}$ extended by $e$ between vertices $v_{1}$ and $v_{2}$, and vertices $w_{1}$, $w_{2}$ of $G_{1}$. Suppose $e \notin$ the edges of $G_{2}$ and $w_{1}=v_{1}$ and $w_{2}=v_{2}$. Then $w_{2} \in G_{1}$.reachableFrom $\left(w_{1}\right)$.

(42) Let us consider a graph $G_{2}$, vertices $v_{1}, v_{2}$ of $G_{2}$, an object $e$, a supergraph $G_{1}$ of $G_{2}$ extended by $e$ between vertices $v_{1}$ and $v_{2}$, and a vertex $w_{1}$ of $G_{1}$. Suppose $e \notin$ the edges of $G_{2}$ and $w_{1}=v_{1}$. Then $G_{1}$.reachableFrom $\left(w_{1}\right)=\left(G_{2}\right.$.reachableFrom $\left.\left(v_{1}\right)\right) \cup\left(G_{2}\right.$.reachableFrom $\left.\left(v_{2}\right)\right)$.

Proof: For every object $x$ such that $x \in G_{1}$.reachableFrom $\left(w_{1}\right)$ holds $x$ $\in\left(G_{2}\right.$.reachableFrom $\left.\left(v_{1}\right)\right) \cup\left(G_{2}\right.$.reachableFrom $\left.\left(v_{2}\right)\right)$. $G_{2}$.reachableFrom $\left(v_{2}\right) \subseteq G_{1}$.reachableFrom $\left(w_{1}\right)$.

(43) Let us consider a graph $G_{2}$, vertices $v_{1}, v_{2}$ of $G_{2}$, an object $e$, a supergraph $G_{1}$ of $G_{2}$ extended by $e$ between vertices $v_{1}$ and $v_{2}$, a vertex $w$ of $G_{1}$, and a vertex $v$ of $G_{2}$. Suppose $e \notin$ the edges of $G_{2}$ and $v=w$ and $v \notin G_{2}$.reachableFrom $\left(v_{1}\right)$ and $v \notin G_{2}$.reachableFrom $\left(v_{2}\right)$. Then $G_{1} \cdot \operatorname{reachableFrom}(w)=G_{2}$.reachableFrom $(v)$.

Proof: For every object $x$ such that $x \in G_{1}$.reachableFrom $(w)$ holds $x \in G_{2}$.reachableFrom $(v)$.

(44) Let us consider a graph $G_{2}$, vertices $v_{1}, v_{2}$ of $G_{2}$, an object $e$, and a supergraph $G_{1}$ of $G_{2}$ extended by $e$ between vertices $v_{1}$ and $v_{2}$. Suppose $e \notin$ the edges of $G_{2}$. Then $G_{1}$.componentSet ()$=\left(G_{2} \cdot \operatorname{componentSet}() \backslash\right.$ $\left\{G_{2}\right.$.reachableFrom $\left(v_{1}\right), G_{2}$.reachableFrom $\left.\left.\left(v_{2}\right)\right\}\right) \cup\left\{\left(G_{2}\right.\right.$.reachableFrom $\left.\left(v_{1}\right)\right) \cup\left(G_{2}\right.$.reachableFrom $\left.\left.\left(v_{2}\right)\right)\right\}$. 
(45) Let us consider a graph $G_{1}$, a vertex $v$ of $G_{1}$, and a subgraph $G_{2}$ of $G_{1}$ with vertex $v$ removed. If $v$ is endvertex, then $G_{1}$.numComponents ()$=$ $G_{2}$.numComponents().

Proof: $G_{1}$ is not trivial. There exists a function $f$ such that $f$ is oneto-one and $\operatorname{dom} f=G_{1}$.componentSet() and $\operatorname{rng} f=G_{2}$.componentSet().

Let $G$ be a graph. One can check that every vertex of $G$ which is endvertex is also non cut-vertex. Now we state the propositions:

(46) Let us consider a non trivial, finite, connected graph $G_{1}$, and a non spanning, connected subgraph $G_{2}$ of $G_{1}$. Then there exists a vertex $v$ of $G_{1}$ such that

(i) $v$ is not cut-vertex, and

(ii) $v \notin$ the vertices of $G_{2}$.

Proof: Define $\mathcal{P}$ [natural number] $\equiv$ for every non trivial, finite, connected graph $G_{1}$ for every non spanning, connected subgraph $G_{2}$ of $G_{1}$ such that $G_{1}$.order ()$+\$_{1}=G_{1} \cdot \operatorname{size}()+1$ there exists a vertex $v$ of $G_{1}$ such that $v$ is not cut-vertex and $v \notin$ the vertices of $G_{2} . \mathcal{P}[0]$. For every natural number $k$ such that $\mathcal{P}[k]$ holds $\mathcal{P}[k+1]$. For every natural number $k, \mathcal{P}[k]$.

(47) Let us consider a non trivial, simple graph $G_{1}$, a vertex $v$ of $G_{1}$, and a subgraph $G_{2}$ of $G_{1}$ with vertex $v$ removed. Then $G_{1}$ is a supergraph of $G_{2}$ extended by vertex $v$ and edges between $v$ and $v$.allNeighbors() of $G_{2}$.

\section{Edgeless and Non Edgeless Graphs}

Let $G$ be a graph. We say that $G$ is edgeless if and only if

(Def. 1) the edges of $G=\emptyset$.

Let us consider a graph $G$. Now we state the propositions:

(48) $G$ is edgeless if and only if $\overline{\bar{\alpha}}=0$, where $\alpha$ is the edges of $G$.

(49) $G$ is edgeless if and only if $G \cdot \operatorname{size}()=0$.

Let $G$ be a graph. Observe that every subgraph of $G$ with edges the edges of $G$ removed is edgeless and there exists a graph which is edgeless and there exists a subgraph of $G$ which is edgeless and spanning and there exists a subgraph of $G$ which is edgeless and trivial.

Let $G$ be an edgeless graph. One can check that the edges of $G$ is empty and every graph which is edgeless is also non-multi, non-directed-multi, loopless, simple, and directed-simple and every graph which is trivial and loopless is also edgeless. 
Let $V$ be a non empty set and $S, T$ be functions from $\emptyset$ into $V$. One can check that createGraph $(V, \emptyset, S, T)$ is edgeless.

Now we state the propositions:

(50) Let us consider an edgeless graph $G$, and objects $e, v_{1}, v_{2}$. Then

(i) $e$ does not join $v_{1}$ and $v_{2}$ in $G$, and

(ii) $e$ does not join $v_{1}$ to $v_{2}$ in $G$.

(51) Let us consider an edgeless graph $G$, an object $e$, and sets $X, Y$. Then

(i) $e$ does not join a vertex from $X$ and a vertex from $Y$ in $G$, and

(ii) $e$ does not join a vertex from $X$ to a vertex from $Y$ in $G$.

(52) Let us consider graphs $G_{1}, G_{2}$. If $G_{1} \approx G_{2}$, then if $G_{1}$ is edgeless, then $G_{2}$ is edgeless.

Let $G$ be an edgeless graph. Let us observe that every walk of $G$ is trivial and every subgraph of $G$ is edgeless.

Let $X$ be a set. Note that $G$.edgesInto $(X)$ is empty and $G$.edgesOutOf $(X)$ is empty and $G$.edgesInOut $(X)$ is empty and $G$.edgesBetween $(X)$ is empty and $G$.set(WeightSelector, $X$ ) is edgeless and $G$.set(ELabelSelector, $X$ ) is edgeless and $G$.set(VLabelSelector, $X$ ) is edgeless and every supergraph of $G$ extended by the vertices from $X$ is edgeless and every graph given by reversing directions of the edges $X$ of $G$ is edgeless.

Let $Y$ be a set. Let us note that $G$.edgesBetween $(X, Y)$ is empty and

$G$.edgesDBetween $(X, Y)$ is empty and every graph which is edgeless is also acyclic and chordal and every graph which is trivial and edgeless is also tree-like and every graph which is non trivial and edgeless is also non connected, non tree-like, and non complete and every graph which is connected and edgeless is also trivial.

Now we state the propositions:

(53) Let us consider an edgeless graph $G_{1}$, and a subgraph $G_{2}$ of $G_{1}$. Then $G_{1}$ is a supergraph of $G_{2}$ extended by the vertices from (the vertices of $\left.G_{1}\right) \backslash$ (the vertices of $G_{2}$ ). The theorem is a consequence of (33).

(54) Let us consider a graph $G_{2}$, vertices $v_{1}, v_{2}$ of $G_{2}$, an object $e$, and a supergraph $G_{1}$ of $G_{2}$ extended by $e$ between vertices $v_{1}$ and $v_{2}$. Suppose $e \notin$ the edges of $G_{2}$. Then $G_{1}$ is not edgeless.

(55) Let us consider a graph $G_{2}$, a vertex $v_{1}$ of $G_{2}$, objects $e, v_{2}$, and a supergraph $G_{1}$ of $G_{2}$ extended by $v_{1}, v_{2}$ and $e$ between them. Suppose $v_{2} \notin$ the vertices of $G_{2}$ and $e \notin$ the edges of $G_{2}$. Then $G_{1}$ is not edgeless.

(56) Let us consider a graph $G_{2}$, objects $v_{1}, e$, a vertex $v_{2}$ of $G_{2}$, and a supergraph $G_{1}$ of $G_{2}$ extended by $v_{1}, v_{2}$ and $e$ between them. Suppose 
$v_{1} \notin$ the vertices of $G_{2}$ and $e \notin$ the edges of $G_{2}$. Then $G_{1}$ is not edgeless.

(57) Let us consider a graph $G_{2}$, an object $v$, a non empty subset $V$ of the vertices of $G_{2}$, and a supergraph $G_{1}$ of $G_{2}$ extended by vertex $v$ and edges between $v$ and $V$ of $G_{2}$. Suppose $v \notin$ the vertices of $G_{2}$. Then $G_{1}$ is not edgeless.

Let $G$ be a graph. Let us observe that every supergraph of $G$ extended by vertex the vertices of $G$ and edges from the vertices of $G$ to the vertices of $G$ is non edgeless and every supergraph of $G$ extended by vertex the vertices of $G$ and edges from the vertices of $G$ to the vertices of $G$ is non edgeless and every supergraph of $G$ extended by vertex the vertices of $G$ and edges between the vertices of $G$ and the vertices of $G$ is non edgeless.

Let $v$ be a vertex of $G$. Let us note that every supergraph of $G$ extended by $v$, the vertices of $G$ and the edges of $G$ between them is non edgeless and every supergraph of $G$ extended by the vertices of $G, v$ and the edges of $G$ between them is non edgeless.

Let $w$ be a vertex of $G$. Let us note that every supergraph of $G$ extended by the edges of $G$ between vertices $v$ and $w$ is non edgeless.

Let $G$ be an edgeless graph. Note that every component of $G$ is trivial and $v$.edgesIn() is empty and $v$.edgesOut() is empty and $v$.edgesInOut() is empty and every vertex of $G$ is isolated, non cut-vertex, and non endvertex and $v$.inDegree() is empty and $v$.outDegree() is empty and $v$.inNeighbors () is empty and $v$.outNeighbors () is empty and $v$.degree () is empty and $v$.allNeighbors () is empty and there exists a graph which is trivial, finite, and edgeless and there exists a graph which is non trivial, finite, and edgeless and there exists a graph which is trivial, finite, and non edgeless and there exists a graph which is non trivial, finite, and non edgeless.

Let $G$ be a non edgeless graph. One can check that the edges of $G$ is non empty and every supergraph of $G$ is non edgeless.

Let $X$ be a set. One can verify that every graph given by reversing directions of the edges $X$ of $G$ is non edgeless and $G$.set(WeightSelector, $X$ ) is non edgeless and $G$.set(ELabelSelector, $X$ ) is non edgeless and $G$.set(VLabelSelector, $X$ ) is non edgeless.

An edge of $G$ is an element of the edges of $G$. Now we state the proposition:

(58) Let us consider a finite, edgeless graph $G_{1}$, and a subgraph $G_{2}$ of $G_{1}$. If $G_{1}$.order ()$=G_{2}$.order(), then $G_{1} \approx G_{2}$.

Let $F$ be a graph-yielding function. We say that $F$ is edgeless if and only if

(Def. 2) for every object $x$ such that $x \in \operatorname{dom} F$ there exists a graph $G$ such that $F(x)=G$ and $G$ is edgeless. 
Let $F$ be a non empty, graph-yielding function. Note that $F$ is edgeless if and only if the condition (Def. 3) is satisfied.

(Def. 3) for every element $x$ of $\operatorname{dom} F, F(x)$ is edgeless.

Let $S$ be a graph sequence. Let us note that $S$ is edgeless if and only if the condition (Def. 4) is satisfied.

(Def. 4) for every natural number $n, S(n)$ is edgeless.

Let us observe that every graph-yielding function which is trivial and loopless is also edgeless and every graph-yielding function which is edgeless is also nonmulti, non-directed-multi, loopless, simple, directed-simple, and acyclic.

Let $F$ be an edgeless, non empty, graph-yielding function and $x$ be an element of $\operatorname{dom} F$. Observe that $F(x)$ is edgeless.

Let $S$ be an edgeless graph sequence and $x$ be a natural number. Observe that $S(x)$ is edgeless.

\section{Finite Graph Sequences}

Let $G$ be a graph. Note that $\langle G\rangle$ is graph-yielding.

Let $G$ be a finite graph. Let us note that $\langle G\rangle$ is finite.

Let $G$ be a loopless graph. Observe that $\langle G\rangle$ is loopless.

Let $G$ be a trivial graph. Let us observe that $\langle G\rangle$ is trivial.

Let $G$ be a non trivial graph. Let us observe that $\langle G\rangle$ is nontrivial.

Let $G$ be a non-multi graph. One can verify that $\langle G\rangle$ is non-multi.

Let $G$ be a non-directed-multi graph. One can check that $\langle G\rangle$ is non-directedmulti.

Let $G$ be a simple graph. Note that $\langle G\rangle$ is simple.

Let $G$ be a directed-simple graph. Let us note that $\langle G\rangle$ is directed-simple.

Let $G$ be a connected graph. Observe that $\langle G\rangle$ is connected.

Let $G$ be an acyclic graph. Let us observe that $\langle G\rangle$ is acyclic.

Let $G$ be a tree-like graph. One can verify that $\langle G\rangle$ is tree-like.

Let $G$ be an edgeless graph. One can check that $\langle G\rangle$ is edgeless and there exists a finite sequence which is empty and graph-yielding and there exists a finite sequence which is non empty and graph-yielding.

Let $p$ be a non empty, graph-yielding finite sequence. Note that $p(1)$ is function-like and relation-like and $p(\operatorname{len} p)$ is function-like and relation-like and $p(1)$ is finite and $\mathbb{N}$-defined and $p(\operatorname{len} p)$ is finite and $\mathbb{N}$-defined and $p(1)$ is graphlike and $p(\operatorname{len} p)$ is graph-like and there exists a graph-yielding finite sequence which is non empty, finite, loopless, trivial, non-multi, non-directed-multi, simple, directed-simple, connected, acyclic, tree-like, and edgeless and there exists a graph-yielding finite sequence which is non empty, finite, loopless, nontrivial, 
non-multi, non-directed-multi, simple, directed-simple, connected, acyclic, and tree-like.

Let $p$ be a graph-yielding finite sequence and $n$ be a natural number. Let us observe that $p\left\lceil n\right.$ is graph-yielding and $p_{\lfloor n}$ is graph-yielding.

Let $m$ be a natural number. Note that $\operatorname{smid}(p, m, n)$ is graph-yielding and $\langle p(m), \ldots, p(n)\rangle$ is graph-yielding.

Let $p$ be a finite, graph-yielding finite sequence. One can verify that $p\lceil n$ is finite and $p_{\lfloor n}$ is finite and $\operatorname{smid}(p, m, n)$ is finite and $\langle p(m), \ldots, p(n)\rangle$ is finite.

Let $p$ be a loopless, graph-yielding finite sequence. One can verify that $p\lceil n$ is loopless and $p_{\lfloor n}$ is loopless and $\operatorname{smid}(p, m, n)$ is loopless and $\langle p(m), \ldots, p(n)\rangle$ is loopless.

Let $p$ be a trivial, graph-yielding finite sequence. One can verify that $p\lceil n$ is trivial and $p_{\lfloor n}$ is trivial and $\operatorname{smid}(p, m, n)$ is trivial and $\langle p(m), \ldots, p(n)\rangle$ is trivial.

Let $p$ be a nontrivial, graph-yielding finite sequence. One can verify that $p\lceil n$ is nontrivial and $p_{l n}$ is nontrivial and $\operatorname{smid}(p, m, n)$ is nontrivial and

$\langle p(m), \ldots, p(n)\rangle$ is nontrivial.

Let $p$ be a non-multi, graph-yielding finite sequence. One can verify that $p\lceil n$ is non-multi and $p_{l n}$ is non-multi and $\operatorname{smid}(p, m, n)$ is non-multi and

$\langle p(m), \ldots, p(n)\rangle$ is non-multi.

Let $p$ be a non-directed-multi, graph-yielding finite sequence. One can verify that $p\left\lceil n\right.$ is non-directed-multi and $p_{\lfloor n}$ is non-directed-multi and $\operatorname{smid}(p, m, n)$ is non-directed-multi and $\langle p(m), \ldots, p(n)\rangle$ is non-directed-multi.

Let $p$ be a simple, graph-yielding finite sequence. One can verify that $p\lceil n$ is simple and $p_{l n}$ is simple and $\operatorname{smid}(p, m, n)$ is simple and $\langle p(m), \ldots, p(n)\rangle$ is simple.

Let $p$ be a directed-simple, graph-yielding finite sequence. One can verify that $p\left\lceil n\right.$ is directed-simple and $p_{\lfloor n}$ is directed-simple and $\operatorname{smid}(p, m, n)$ is directedsimple and $\langle p(m), \ldots, p(n)\rangle$ is directed-simple.

Let $p$ be a connected, graph-yielding finite sequence. One can verify that $p\lceil n$ is connected and $p_{l n}$ is connected and $\operatorname{smid}(p, m, n)$ is connected and

$\langle p(m), \ldots, p(n)\rangle$ is connected.

Let $p$ be an acyclic, graph-yielding finite sequence. One can verify that $p\lceil n$ is acyclic and $p_{\lfloor n}$ is acyclic and $\operatorname{smid}(p, m, n)$ is acyclic and $\langle p(m), \ldots, p(n)\rangle$ is acyclic.

Let $p$ be a tree-like, graph-yielding finite sequence. One can verify that $p\lceil n$ is tree-like and $p_{\lfloor n}$ is tree-like and $\operatorname{smid}(p, m, n)$ is tree-like and $\langle p(m), \ldots, p(n)\rangle$ is tree-like.

Let $p$ be an edgeless, graph-yielding finite sequence. One can verify that $p\lceil n$ is edgeless and $p_{l n}$ is edgeless and $\operatorname{smid}(p, m, n)$ is edgeless and $\langle p(m), \ldots, p(n)\rangle$ 
is edgeless.

Let $p, q$ be graph-yielding finite sequences. Let us note that $p^{\frown} q$ is graphyielding and $p \curvearrowright q$ is graph-yielding.

Let $p, q$ be finite, graph-yielding finite sequences. Let us observe that $p^{\frown} q$ is finite and $p \curvearrowright q$ is finite.

Let $p, q$ be loopless, graph-yielding finite sequences. Let us observe that $p^{\frown} q$ is loopless and $p \curvearrowright q$ is loopless.

Let $p, q$ be trivial, graph-yielding finite sequences. Let us observe that $p^{\frown} q$ is trivial and $p \curvearrowright q$ is trivial.

Let $p, q$ be nontrivial, graph-yielding finite sequences. Let us observe that $p^{\frown} q$ is nontrivial and $p \propto q$ is nontrivial.

Let $p, q$ be non-multi, graph-yielding finite sequences. Observe that $p^{\frown} q$ is non-multi and $p \wedge q$ is non-multi.

Let $p, q$ be non-directed-multi, graph-yielding finite sequences. Observe that $p^{\frown} q$ is non-directed-multi and $p \curvearrowright q$ is non-directed-multi.

Let $p, q$ be simple, graph-yielding finite sequences. Observe that $p^{\frown} q$ is simple and $p \propto q$ is simple.

Let $p, q$ be directed-simple, graph-yielding finite sequences. One can verify that $p^{\frown} q$ is directed-simple and $p \curvearrowright q$ is directed-simple.

Let $p, q$ be connected, graph-yielding finite sequences. Note that $p^{\frown} q$ is connected and $p \wedge q$ is connected.

Let $p, q$ be acyclic, graph-yielding finite sequences. Note that $p^{\frown} q$ is acyclic and $p \propto q$ is acyclic.

Let $p, q$ be tree-like, graph-yielding finite sequences. Note that $p^{\frown} q$ is treelike and $p \curvearrowright q$ is tree-like.

Let $p, q$ be edgeless, graph-yielding finite sequences. Observe that $p^{\frown} q$ is edgeless and $p \wedge q$ is edgeless.

Let $G_{1}, G_{2}$ be graphs. Note that $\left\langle G_{1}, G_{2}\right\rangle$ is graph-yielding.

Let $G_{3}$ be a graph. Let us note that $\left\langle G_{1}, G_{2}, G_{3}\right\rangle$ is graph-yielding.

Let $G_{1}, G_{2}$ be finite graphs. Let us observe that $\left\langle G_{1}, G_{2}\right\rangle$ is finite.

Let $G_{3}$ be a finite graph. One can verify that $\left\langle G_{1}, G_{2}, G_{3}\right\rangle$ is finite.

Let $G_{1}, G_{2}$ be loopless graphs. Note that $\left\langle G_{1}, G_{2}\right\rangle$ is loopless.

Let $G_{3}$ be a loopless graph. Let us note that $\left\langle G_{1}, G_{2}, G_{3}\right\rangle$ is loopless.

Let $G_{1}, G_{2}$ be trivial graphs. Let us observe that $\left\langle G_{1}, G_{2}\right\rangle$ is trivial.

Let $G_{3}$ be a trivial graph. One can verify that $\left\langle G_{1}, G_{2}, G_{3}\right\rangle$ is trivial.

Let $G_{1}, G_{2}$ be non trivial graphs. One can check that $\left\langle G_{1}, G_{2}\right\rangle$ is nontrivial.

Let $G_{3}$ be a non trivial graph. One can check that $\left\langle G_{1}, G_{2}, G_{3}\right\rangle$ is nontrivial.

Let $G_{1}, G_{2}$ be non-multi graphs. Let us note that $\left\langle G_{1}, G_{2}\right\rangle$ is non-multi.

Let $G_{3}$ be a non-multi graph. Observe that $\left\langle G_{1}, G_{2}, G_{3}\right\rangle$ is non-multi. 
Let $G_{1}, G_{2}$ be non-directed-multi graphs. One can verify that $\left\langle G_{1}, G_{2}\right\rangle$ is non-directed-multi.

Let $G_{3}$ be a non-directed-multi graph. One can check that $\left\langle G_{1}, G_{2}, G_{3}\right\rangle$ is non-directed-multi.

Let $G_{1}, G_{2}$ be simple graphs. Let us note that $\left\langle G_{1}, G_{2}\right\rangle$ is simple.

Let $G_{3}$ be a simple graph. Observe that $\left\langle G_{1}, G_{2}, G_{3}\right\rangle$ is simple.

Let $G_{1}, G_{2}$ be directed-simple graphs. One can verify that $\left\langle G_{1}, G_{2}\right\rangle$ is directedsimple.

Let $G_{3}$ be a directed-simple graph. One can check that $\left\langle G_{1}, G_{2}, G_{3}\right\rangle$ is directed-simple.

Let $G_{1}, G_{2}$ be connected graphs. Let us note that $\left\langle G_{1}, G_{2}\right\rangle$ is connected.

Let $G_{3}$ be a connected graph. Observe that $\left\langle G_{1}, G_{2}, G_{3}\right\rangle$ is connected.

Let $G_{1}, G_{2}$ be acyclic graphs. One can verify that $\left\langle G_{1}, G_{2}\right\rangle$ is acyclic.

Let $G_{3}$ be an acyclic graph. One can check that $\left\langle G_{1}, G_{2}, G_{3}\right\rangle$ is acyclic.

Let $G_{1}, G_{2}$ be tree-like graphs. Let us note that $\left\langle G_{1}, G_{2}\right\rangle$ is tree-like.

Let $G_{3}$ be a tree-like graph. Observe that $\left\langle G_{1}, G_{2}, G_{3}\right\rangle$ is tree-like.

Let $G_{1}, G_{2}$ be edgeless graphs. One can verify that $\left\langle G_{1}, G_{2}\right\rangle$ is edgeless.

Let $G_{3}$ be an edgeless graph. One can check that $\left\langle G_{1}, G_{2}, G_{3}\right\rangle$ is edgeless.

\section{Construction of Finite Graphs}

Now we state the propositions:

(59) Let us consider a graph $G_{2}$, a finite set $V$, and a supergraph $G_{1}$ of $G_{2}$ extended by the vertices from $V$. Then there exists a non empty, graphyielding finite sequence $p$ such that

(i) $p(1) \approx G_{2}$, and

(ii) $p(\operatorname{len} p)=G_{1}$, and

(iii) len $p=\overline{\overline{V \backslash \alpha}}+1$, and

(iv) for every element $n$ of $\operatorname{dom} p$ such that $n \leqslant \operatorname{len} p-1$ there exists a vertex $v$ of $G_{1}$ such that $p(n+1)$ is a supergraph of $p(n)$ extended by $v$ and $v \notin$ the vertices of $p(n)$,

where $\alpha$ is the vertices of $G_{2}$.

Proof: Define $\mathcal{P}$ [natural number] $\equiv$ for every finite set $V$ for every supergraph $G_{1}$ of $G_{2}$ extended by the vertices from $V$ such that

$\overline{\overline{V \backslash\left(\text { the vertices of } G_{2}\right)}}=\$_{1}$ there exists a non empty, graph-yielding finite sequence $p$ such that $p(1) \approx G_{2}$ and $p(\operatorname{len} p)=G_{1}$ and $\operatorname{len} p=$ $\overline{\overline{V \backslash\left(\text { the vertices of } G_{2}\right)}}+1$ and for every element $n$ of $\operatorname{dom} p$ such that 
$n \leqslant \operatorname{len} p-1$ there exists a vertex $v$ of $G_{1}$ such that $p(n+1)$ is a supergraph of $p(n)$ extended by $v$ and $v \notin$ the vertices of $p(n)$.

$\mathcal{P}[0]$. For every natural number $k$ such that $\mathcal{P}[k]$ holds $\mathcal{P}[k+1]$. For every natural number $k, \mathcal{P}[k]$.

(60) Let us consider a finite graph $G$, and a subgraph $H$ of $G$. Suppose $G \cdot \operatorname{size}()=H \cdot \operatorname{size}()$. Then there exists a non empty, finite, graph-yielding finite sequence $p$ such that

(i) $p(1) \approx H$, and

(ii) $p(\operatorname{len} p)=G$, and

(iii) len $p=G$.order ()$-H$.order ()$+1$, and

(iv) for every element $n$ of $\operatorname{dom} p$ such that $n \leqslant \operatorname{len} p-1$ there exists a vertex $v$ of $G$ such that $p(n+1)$ is a supergraph of $p(n)$ extended by $v$ and $v \notin$ the vertices of $p(n)$.

Proof: Set $V=($ the vertices of $G) \backslash($ the vertices of $H) . G$ is a supergraph of $H$ extended by the vertices from $V$. Consider $p$ being a non empty, graph-yielding finite sequence such that $p(1) \approx H$ and $p(\operatorname{len} p)=G$ and len $p=\overline{\overline{V \backslash \alpha}}+1$, where $\alpha$ is the vertices of $H$ and for every element $n$ of $\operatorname{dom} p$ such that $n \leqslant \operatorname{len} p-1$ there exists a vertex $v$ of $G$ such that $p(n+1)$ is a supergraph of $p(n)$ extended by $v$ and $v \notin$ the vertices of $p(n)$.

Define $\mathcal{P}$ [natural number] $\equiv$ for every element $n$ of $\operatorname{dom} p$ such that $\$_{1}=n$ holds $p(n)$ is finite. For every non zero natural number $k$ such that $\mathcal{P}[k]$ holds $\mathcal{P}[k+1]$. For every non zero natural number $k, \mathcal{P}[k]$. For every element $x$ of $\operatorname{dom} p, p(x)$ is finite.

(61) Let us consider a finite, edgeless graph $G$, and a subgraph $H$ of $G$. Then there exists a non empty, finite, edgeless, graph-yielding finite sequence $p$ such that

(i) $p(1) \approx H$, and

(ii) $p(\operatorname{len} p)=G$, and

(iii) len $p=G$.order ()$-H$.order ()$+1$, and

(iv) for every element $n$ of $\operatorname{dom} p \operatorname{such}$ that $n \leqslant \operatorname{len} p-1$ there exists a vertex $v$ of $G$ such that $p(n+1)$ is a supergraph of $p(n)$ extended by $v$ and $v \notin$ the vertices of $p(n)$.

Proof: G.size() $=0$. Consider $p$ being a non empty, finite, graphyielding finite sequence such that $p(1) \approx H$ and $p(\operatorname{len} p)=G$ and $\operatorname{len} p=$ $G$.order ()$-H$.order ()$+1$ and for every element $n$ of $\operatorname{dom} p$ such that 
$n \leqslant \operatorname{len} p-1$ there exists a vertex $v$ of $G$ such that $p(n+1)$ is a supergraph of $p(n)$ extended by $v$ and $v \notin$ the vertices of $p(n)$. Define $\mathcal{P}$ [natural number] $\equiv$ for every element $n$ of $\operatorname{dom} p$ such that $\$_{1}=n$ holds $p(n)$ is edgeless.

$\mathcal{P}[1]$. For every non zero natural number $k$ such that $\mathcal{P}[k]$ holds $\mathcal{P}[k+$ $1]$. For every non zero natural number $k, \mathcal{P}[k]$. For every element $x$ of $\operatorname{dom} p, p(x)$ is edgeless.

(62) Let us consider a finite, edgeless graph $G$. Then there exists a non empty, finite, edgeless, graph-yielding finite sequence $p$ such that

(i) $p(1)$ is trivial and edgeless, and

(ii) $p(\operatorname{len} p)=G$, and

(iii) len $p=G$.order(), and

(iv) for every element $n$ of $\operatorname{dom} p$ such that $n \leqslant \operatorname{len} p-1$ there exists a vertex $v$ of $G$ such that $p(n+1)$ is a supergraph of $p(n)$ extended by $v$ and $v \notin$ the vertices of $p(n)$.

The theorem is a consequence of (61) and (52).

The scheme FinEdgelessGraphs deals with a unary predicate $\mathcal{P}$ and states that

(Sch. 1) For every finite, edgeless graph $G, \mathcal{P}[G]$

provided

- for every trivial, edgeless graph $G, \mathcal{P}[G]$ and

- for every finite, edgeless graph $G_{2}$ and for every object $v$ and for every supergraph $G_{1}$ of $G_{2}$ extended by $v$ such that $v \notin$ the vertices of $G_{2}$ and $\mathcal{P}\left[G_{2}\right]$ holds $\mathcal{P}\left[G_{1}\right]$.

Now we state the propositions:

(63) Let us consider a non empty, graph-yielding finite sequence $p$. Suppose $p(1)$ is edgeless and for every element $n$ of $\operatorname{dom} p$ such that $n \leqslant \operatorname{len} p-1$ there exists an object $v$ such that $p(n+1)$ is a supergraph of $p(n)$ extended by $v$. Then $p($ len $p)$ is edgeless.

Proof: Define $\mathcal{P}$ [natural number] $\equiv$ for every non empty, graph-yielding finite sequence $p$ such that len $p=\$_{1}$ and $p(1)$ is edgeless and for every element $n$ of $\operatorname{dom} p$ such that $n \leqslant$ len $p-1$ there exists an object $v$ such that $p(n+1)$ is a supergraph of $p(n)$ extended by $v$ holds $p(\operatorname{len} p)$ is edgeless.

For every non zero natural number $m$ such that $\mathcal{P}[m]$ holds $\mathcal{P}[m+1]$. For every non zero natural number $m, \mathcal{P}[m]$.

(64) Let us consider a finite graph $G$, and a spanning subgraph $H$ of $G$. Then there exists a non empty, finite, graph-yielding finite sequence $p$ such that 
(i) $p(1) \approx H$, and

(ii) $p($ len $p)=G$, and

(iii) len $p=G$.size ()$-H \cdot \operatorname{size}()+1$, and

(iv) for every element $n$ of $\operatorname{dom} p$ such that $n \leqslant \operatorname{len} p-1$ there exist vertices $v_{1}, v_{2}$ of $G$ and there exists an object $e$ such that $p(n+1)$ is a supergraph of $p(n)$ extended by $e$ between vertices $v_{1}$ and $v_{2}$ and $e \in$ (the edges of $G) \backslash$ (the edges of $p(n)$ ) and $v_{1}, v_{2} \in$ the vertices of $p(n)$.

Proof: Define $\mathcal{P}$ [natural number] $\equiv$ for every spanning subgraph $H$ of $G$ such that $G$.size ()$-H$.size ()$=\$_{1}$ there exists a non empty, finite, graph-yielding finite sequence $p$ such that $p(1) \approx H$ and $p(\operatorname{len} p)=G$ and len $p=G$.size ()$-H$.size ()$+1$ and for every element $n$ of $\operatorname{dom} p$ such that $n \leqslant \operatorname{len} p-1$ there exist vertices $v_{1}, v_{2}$ of $G$ and there exists an object $e$ such that $p(n+1)$ is a supergraph of $p(n)$ extended by $e$ between vertices $v_{1}$ and $v_{2}$ and $e \in$ (the edges of $G$ ) \(the edges of $p(n)$ ) and $v_{1}, v_{2} \in$ the vertices of $p(n)$.

$\mathcal{P}[0]$. For every natural number $k$ such that $\mathcal{P}[k]$ holds $\mathcal{P}[k+1]$. For every natural number $k, \mathcal{P}[k]$.

(65) Let us consider a finite graph $G$. Then there exists a non empty, finite, graph-yielding finite sequence $p$ such that

(i) $p(1)$ is edgeless, and

(ii) $p(\operatorname{len} p)=G$, and

(iii) len $p=G$.size ()$+1$, and

(iv) for every element $n$ of $\operatorname{dom} p$ such that $n \leqslant \operatorname{len} p-1$ there exist vertices $v_{1}, v_{2}$ of $G$ and there exists an object $e$ such that $p(n+1)$ is a supergraph of $p(n)$ extended by $e$ between vertices $v_{1}$ and $v_{2}$ and $e \in($ the edges of $G) \backslash$ (the edges of $p(n))$ and $v_{1}, v_{2} \in$ the vertices of $p(n)$.

The theorem is a consequence of (64), (52), and (49).

(66) Let us consider a finite, connected graph $G$, and a spanning, connected subgraph $H$ of $G$. Then there exists a non empty, finite, connected, graphyielding finite sequence $p$ such that

(i) $p(1) \approx H$, and

(ii) $p(\operatorname{len} p)=G$, and

(iii) $\operatorname{len} p=G \cdot \operatorname{size}()-H \cdot \operatorname{size}()+1$, and 
(iv) for every element $n$ of $\operatorname{dom} p$ such that $n \leqslant \operatorname{len} p-1$ there exist vertices $v_{1}, v_{2}$ of $G$ and there exists an object $e$ such that $p(n+1)$ is a supergraph of $p(n)$ extended by $e$ between vertices $v_{1}$ and $v_{2}$ and $e \in($ the edges of $G) \backslash$ (the edges of $p(n))$ and $v_{1}, v_{2} \in$ the vertices of $p(n)$.

Proof: Consider $p$ being a non empty, finite, graph-yielding finite sequence such that $p(1) \approx H$ and $p(\operatorname{len} p)=G$ and len $p=G \cdot \operatorname{size}()-H \cdot \operatorname{size}()+1$ and for every element $n$ of $\operatorname{dom} p$ such that $n \leqslant \operatorname{len} p-1$ there exist vertices $v_{1}, v_{2}$ of $G$ and there exists an object $e$ such that $p(n+1)$ is a supergraph of $p(n)$ extended by $e$ between vertices $v_{1}$ and $v_{2}$ and $e \in$ (the edges of $G) \backslash($ the edges of $p(n))$ and $v_{1}, v_{2} \in$ the vertices of $p(n)$.

Define $\mathcal{P}$ [natural number] $\equiv$ for every element $n$ of $\operatorname{dom} p$ such that $\$_{1}=n$ holds $p(n)$ is connected. For every non zero natural number $k$ such that $\mathcal{P}[k]$ holds $\mathcal{P}[k+1]$. For every non zero natural number $k, \mathcal{P}[k]$. For every element $x$ of $\operatorname{dom} p, p(x)$ is connected.

(67) Let us consider a finite graph $G_{1}$, and a subgraph $H$ of $G_{1}$. Then there exists a spanning subgraph $G_{2}$ of $G_{1}$ and there exists a non empty, finite, graph-yielding finite sequence $p$ such that $H \cdot \operatorname{size}()=G_{2} \cdot \operatorname{size}()$ and $p(1) \approx$ $H$ and $p(\operatorname{len} p)=G_{2}$ and len $p=G_{1}$.order ()$-H$.order ()$+1$ and for every element $n$ of $\operatorname{dom} p$ such that $n \leqslant \operatorname{len} p-1$ there exists a vertex $v$ of $G_{1}$ such that $p(n+1)$ is a supergraph of $p(n)$ extended by $v$ and $v \notin$ the vertices of $p(n)$.

Proof: Set $V=$ (the vertices of $\left.G_{1}\right) \backslash\left(\right.$ the vertices of $H$ ). Set $G_{2}=$ the supergraph of $H$ extended by the vertices from $V$. Consider $p$ being a non empty, graph-yielding finite sequence such that $p(1) \approx H$ and $p(\operatorname{len} p)=G_{2}$ and len $p=\overline{\overline{V \backslash \alpha}}+1$, where $\alpha$ is the vertices of $H$ and for every element $n$ of $\operatorname{dom} p$ such that $n \leqslant \operatorname{len} p-1$ there exists a vertex $v$ of $G_{2}$ such that $p(n+1)$ is a supergraph of $p(n)$ extended by $v$ and $v \notin$ the vertices of $p(n)$.

Define $\mathcal{P}$ [natural number] $\equiv$ for every element $n$ of $\operatorname{dom} p$ such that $\$_{1}=n$ holds $p(n)$ is finite. For every non zero natural number $k$ such that $\mathcal{P}[k]$ holds $\mathcal{P}[k+1]$. For every non zero natural number $k, \mathcal{P}[k]$. For every element $x$ of dom $p, p(x)$ is finite. $G_{2}$ is a subgraph of $G_{1}$. Consider $v$ being a vertex of $G_{2}$ such that $p(n+1)$ is a supergraph of $p(n)$ extended by $v$ and $v \notin$ the vertices of $p(n)$.

(68) Let us consider a finite graph $G$, and a subgraph $H$ of $G$. Then there exists a non empty, finite, graph-yielding finite sequence $p$ such that

(i) $p(1) \approx H$, and

(ii) $p(\operatorname{len} p)=G$, and 
(iii) $\operatorname{len} p=G$.order ()$+G \cdot \operatorname{size}()-(H \cdot \operatorname{order}()+H \cdot \operatorname{size}())+1$, and

(iv) for every element $n$ of $\operatorname{dom} p$ such that $n \leqslant \operatorname{len} p-1$ holds there exist vertices $v_{1}, v_{2}$ of $G$ and there exists an object $e$ such that $p(n+1)$ is a supergraph of $p(n)$ extended by $e$ between vertices $v_{1}$ and $v_{2}$ and $e \in($ the edges of $G) \backslash($ the edges of $p(n))$ and $v_{1}, v_{2} \in$ the vertices of $p(n)$ or there exists a vertex $v$ of $G$ such that $p(n+1)$ is a supergraph of $p(n)$ extended by $v$ and $v \notin$ the vertices of $p(n)$.

The theorem is a consequence of (67), (64), (36), and (60).

(69) Let us consider a finite graph $G$. Then there exists a non empty, finite, graph-yielding finite sequence $p$ such that

(i) $p(1)$ is trivial and edgeless, and

(ii) $p(\operatorname{len} p)=G$, and

(iii) len $p=G$.order ()$+G \cdot \operatorname{size}()$, and

(iv) for every element $n$ of $\operatorname{dom} p$ such that $n \leqslant \operatorname{len} p-1$ holds there exist vertices $v_{1}, v_{2}$ of $G$ and there exists an object $e$ such that $p(n+1)$ is a supergraph of $p(n)$ extended by $e$ between vertices $v_{1}$ and $v_{2}$ and $e \in($ the edges of $G) \backslash($ the edges of $p(n))$ and $v_{1}, v_{2} \in$ the vertices of $p(n)$ or there exists a vertex $v$ of $G$ such that $p(n+1)$ is a supergraph of $p(n)$ extended by $v$ and $v \notin$ the vertices of $p(n)$.

The theorem is a consequence of (68), (52), and (49).

The scheme FinGraphs deals with a unary predicate $\mathcal{P}$ and states that

(Sch. 2) For every finite graph $G, \mathcal{P}[G]$

provided

- for every trivial, edgeless graph $G, \mathcal{P}[G]$ and

- for every finite graph $G_{2}$ and for every object $v$ and for every supergraph $G_{1}$ of $G_{2}$ extended by $v$ such that $v \notin$ the vertices of $G_{2}$ and $\mathcal{P}\left[G_{2}\right]$ holds $\mathcal{P}\left[G_{1}\right]$ and

- for every finite graph $G_{2}$ and for every vertices $v_{1}, v_{2}$ of $G_{2}$ and for every object $e$ and for every supergraph $G_{1}$ of $G_{2}$ extended by $e$ between vertices $v_{1}$ and $v_{2}$ such that $e \notin$ the edges of $G_{2}$ and $\mathcal{P}\left[G_{2}\right]$ holds $\mathcal{P}\left[G_{1}\right]$.

Now we state the propositions:

(70) Let us consider a non empty, graph-yielding finite sequence $p$. Suppose $p(1)$ is finite and for every element $n$ of dom $p$ such that $n \leqslant \operatorname{len} p-1$ holds there exists an object $v$ such that $p(n+1)$ is a supergraph of $p(n)$ extended by $v$ or there exist objects $v_{1}, e, v_{2}$ such that $p(n+1)$ is a supergraph of $p(n)$ extended by $e$ between vertices $v_{1}$ and $v_{2}$. Then $p(\operatorname{len} p)$ is finite. 
Proof: Define $\mathcal{Q}$ [natural number] $\equiv$ if $\$_{1} \leqslant$ len $p$, then there exists an element $k$ of $\operatorname{dom} p$ such that $\$_{1}=k$ and $p(k)$ is finite. $\mathcal{Q}[1]$. For every non zero natural number $m$ such that $\mathcal{Q}[m]$ holds $\mathcal{Q}[m+1]$.

For every non zero natural number $m, \mathcal{Q}[m]$. Consider $k$ being an element of $\operatorname{dom} p$ such that len $p=k$ and $p(k)$ is finite.

(71) Let us consider a finite, tree-like graph $G$, and a connected subgraph $H$ of $G$. Then there exists a non empty, finite, tree-like, graph-yielding finite sequence $p$ such that

(i) $p(1) \approx H$, and

(ii) $p(\operatorname{len} p)=G$, and

(iii) $\operatorname{len} p=G$.order ()$-H$.order ()$+1$, and

(iv) for every element $n$ of $\operatorname{dom} p$ such that $n \leqslant \operatorname{len} p-1$ there exist vertices $v_{1}, v_{2}$ of $G$ and there exists an object $e$ such that $p(n+1)$ is a supergraph of $p(n)$ extended by $v_{1}, v_{2}$ and $e$ between them and $e \in$ (the edges of $G) \backslash$ (the edges of $p(n)$ ) and $\left(v_{1} \in\right.$ the vertices of $p(n)$ and $v_{2} \notin$ the vertices of $p(n)$ or $v_{1} \notin$ the vertices of $p(n)$ and $v_{2} \in$ the vertices of $\left.p(n)\right)$.

Proof: Define $\mathcal{P}$ [natural number] $\equiv$ for every finite, tree-like graph $G$ for every connected subgraph $H$ of $G$ such that $\$_{1}=G$.order() $-H$.order() there exists a non empty, finite, tree-like, graph-yielding finite sequence $p$ such that $p(1) \approx H$ and $p(\operatorname{len} p)=G$ and len $p=G$.order ()$-H$.order ()$+1$ and for every element $n$ of $\operatorname{dom} p$ such that $n \leqslant \operatorname{len} p-1$ there exist vertices $v_{1}, v_{2}$ of $G$ and there exists an object $e$ such that $p(n+1)$ is a supergraph of $p(n)$ extended by $v_{1}, v_{2}$ and $e$ between them and $e \in$ (the edges of $G) \backslash($ the edges of $p(n))$ and $\left(v_{1} \in\right.$ the vertices of $p(n)$ and $v_{2} \notin$ the vertices of $p(n)$ or $v_{1} \notin$ the vertices of $p(n)$ and $v_{2} \in$ the vertices of $\left.p(n)\right)$.

$\mathcal{P}[0]$. For every natural number $k$ such that $\mathcal{P}[k]$ holds $\mathcal{P}[k+1]$. For every natural number $k, \mathcal{P}[k]$.

(72) Let us consider a finite, tree-like graph $G$. Then there exists a non empty, finite, tree-like, graph-yielding finite sequence $p$ such that

(i) $p(1)$ is trivial and edgeless, and

(ii) $p(\operatorname{len} p)=G$, and

(iii) len $p=G$.order(), and

(iv) for every element $n$ of $\operatorname{dom} p$ such that $n \leqslant \operatorname{len} p-1$ there exist vertices $v_{1}, v_{2}$ of $G$ and there exists an object $e$ such that $p(n+1)$ is a supergraph of $p(n)$ extended by $v_{1}, v_{2}$ and $e$ between them and $e \in$ (the edges of $G) \backslash$ (the edges of $p(n))$ and $\left(v_{1} \in\right.$ the vertices of 
$p(n)$ and $v_{2} \notin$ the vertices of $p(n)$ or $v_{1} \notin$ the vertices of $p(n)$ and $v_{2} \in$ the vertices of $\left.p(n)\right)$.

The theorem is a consequence of (71) and (52).

The scheme FinTrees deals with a unary predicate $\mathcal{P}$ and states that

(Sch. 3) For every finite, tree-like graph $G, \mathcal{P}[G]$

provided

- for every trivial, edgeless graph $G, \mathcal{P}[G]$ and

- for every finite, tree-like graph $G_{2}$ and for every vertex $v$ of $G_{2}$ and for every objects $e, w$ such that $e \notin$ the edges of $G_{2}$ and $w \notin$ the vertices of $G_{2}$ and $\mathcal{P}\left[G_{2}\right]$ holds for every supergraph $G_{1}$ of $G_{2}$ extended by $v, w$ and $e$ between them, $\mathcal{P}\left[G_{1}\right]$ and for every supergraph $G_{1}$ of $G_{2}$ extended by $w, v$ and $e$ between them, $\mathcal{P}\left[G_{1}\right]$.

Now we state the propositions:

(73) Let us consider a non empty, graph-yielding finite sequence $p$. Suppose $p(1)$ is tree-like and for every element $n$ of $\operatorname{dom} p$ such that $n \leqslant \operatorname{len} p-1$ there exist objects $v_{1}, e, v_{2}$ such that $p(n+1)$ is a supergraph of $p(n)$ extended by $v_{1}, v_{2}$ and $e$ between them. Then $p(\operatorname{len} p)$ is tree-like.

Proof: Define $\mathcal{Q}$ [natural number] $\equiv$ if $\$_{1} \leqslant$ len $p$, then there exists an element $k$ of $\operatorname{dom} p$ such that $\$_{1}=k$ and $p(k)$ is tree-like. $\mathcal{Q}[1]$.

For every non zero natural number $m$ such that $\mathcal{Q}[m]$ holds $\mathcal{Q}[m+1]$. For every non zero natural number $m, \mathcal{Q}[m]$. Consider $k$ being an element of $\operatorname{dom} p$ such that len $p=k$ and $p(k)$ is tree-like.

(74) Let us consider a finite, connected graph $G$. Then there exists a non empty, finite, connected, graph-yielding finite sequence $p$ such that

(i) $p(1)$ is trivial and edgeless, and

(ii) $p(\operatorname{len} p)=G$, and

(iii) len $p=G \cdot \operatorname{size}()+1$, and

(iv) for every element $n$ of $\operatorname{dom} p$ such that $n \leqslant \operatorname{len} p-1$ holds there exist vertices $v_{1}, v_{2}$ of $G$ and there exists an object $e$ such that $p(n+1)$ is a supergraph of $p(n)$ extended by $v_{1}, v_{2}$ and $e$ between them and $e \in($ the edges of $G) \backslash$ (the edges of $p(n))$ and $\left(v_{1} \in\right.$ the vertices of $p(n)$ and $v_{2} \notin$ the vertices of $p(n)$ or $v_{1} \notin$ the vertices of $p(n)$ and $v_{2} \in$ the vertices of $\left.p(n)\right)$ or there exist vertices $v_{1}, v_{2}$ of $G$ and there exists an object $e$ such that $p(n+1)$ is a supergraph of $p(n)$ extended by $e$ between vertices $v_{1}$ and $v_{2}$ and $e \in$ (the edges of $G$ ) \(the edges of $p(n))$ and $v_{1}, v_{2} \in$ the vertices of $p(n)$. 
The theorem is a consequence of (72), (66), and (36).

The scheme FinConnectedGraphs deals with a unary predicate $\mathcal{P}$ and states that

(Sch. 4) For every finite, connected graph $G, \mathcal{P}[G]$

provided

- for every trivial, edgeless graph $G, \mathcal{P}[G]$ and

- for every finite, connected graph $G_{2}$ and for every vertex $v$ of $G_{2}$ and for every objects $e, w$ such that $e \notin$ the edges of $G_{2}$ and $w \notin$ the vertices of $G_{2}$ and $\mathcal{P}\left[G_{2}\right]$ holds for every supergraph $G_{1}$ of $G_{2}$ extended by $v, w$ and $e$ between them, $\mathcal{P}\left[G_{1}\right]$ and for every supergraph $G_{1}$ of $G_{2}$ extended by $w, v$ and $e$ between them, $\mathcal{P}\left[G_{1}\right]$ and

- for every finite, connected graph $G_{2}$ and for every vertices $v_{1}, v_{2}$ of $G_{2}$ and for every object $e$ and for every supergraph $G_{1}$ of $G_{2}$ extended by $e$ between vertices $v_{1}$ and $v_{2}$ such that $e \notin$ the edges of $G_{2}$ and $\mathcal{P}\left[G_{2}\right]$ holds $\mathcal{P}\left[G_{1}\right]$.

Now we state the propositions:

(75) Let us consider a non empty, graph-yielding finite sequence $p$. Suppose $p(1)$ is connected and for every element $n$ of $\operatorname{dom} p$ such that $n \leqslant \operatorname{len} p-1$ there exist objects $v_{1}, e, v_{2}$ such that $p(n+1)$ is supergraph of $p(n)$ extended by $v_{1}, v_{2}$ and $e$ between them or supergraph of $p(n)$ extended by $e$ between vertices $v_{1}$ and $v_{2}$. Then $p(\operatorname{len} p)$ is connected.

Proof: Define $\mathcal{Q}$ [natural number] $\equiv$ if $\$_{1} \leqslant$ len $p$, then there exists an element $k$ of $\operatorname{dom} p$ such that $\$_{1}=k$ and $p(k)$ is connected. $\mathcal{Q}[1]$. For every non zero natural number $m$ such that $\mathcal{Q}[m]$ holds $\mathcal{Q}[m+1]$. For every non zero natural number $m, \mathcal{Q}[m]$.

(76) Let us consider a graph $G_{2}$, an object $v$, a set $V_{1}$, a finite set $V_{2}$, and a supergraph $G_{1}$ of $G_{2}$ extended by vertex $v$ and edges between $v$ and $V_{1} \cup V_{2}$ of $G_{2}$. Suppose $V_{1} \cup V_{2} \subseteq$ the vertices of $G_{2}$ and $v \notin$ the vertices of $G_{2}$ and $V_{1}$ misses $V_{2}$. Then there exists a non empty, graph-yielding finite sequence $p$ such that

(i) $p(1)=G_{2}$, and

(ii) $p(\operatorname{len} p)=G_{1}$, and

(iii) $\operatorname{len} p=\overline{\overline{V_{2}}}+2$, and

(iv) $p(2)$ is a supergraph of $G_{2}$ extended by vertex $v$ and edges between $v$ and $V_{1}$ of $G_{2}$, and 
(v) for every element $n$ of $\operatorname{dom} p$ such that $2 \leqslant n \leqslant \operatorname{len} p-1$ there exists a vertex $w$ of $G_{2}$ and there exists an object $e$ such that $e \in$ (the edges of $\left.G_{1}\right) \backslash($ the edges of $p(n))$ and $p(n+1)$ is supergraph of $p(n)$ extended by $e$ between vertices $v$ and $w$ or supergraph of $p(n)$ extended by $e$ between vertices $w$ and $v$.

Proof: Define $\mathcal{P}$ [natural number] $\equiv$ for every finite set $V_{2}$ for every supergraph $G_{1}$ of $G_{2}$ extended by vertex $v$ and edges between $v$ and $V_{1} \cup V_{2}$ of $G_{2}$ such that $V_{1} \cup V_{2} \subseteq$ the vertices of $G_{2}$ and $v \notin$ the vertices of $G_{2}$ and $V_{1}$ misses $V_{2}$ and $\overline{\overline{V_{2}}}=\$ 1$ there exists a non empty, graph-yielding finite sequence $p$ such that $p(1)=G_{2}$ and $p(\operatorname{len} p)=G_{1}$ and len $p=\overline{\overline{V_{2}}}+2$ and $p(2)$ is a supergraph of $G_{2}$ extended by vertex $v$ and edges between $v$ and $V_{1}$ of $G_{2}$ and for every element $n$ of $\operatorname{dom} p$ such that $2 \leqslant n \leqslant \operatorname{len} p-1$.

There exists a vertex $w$ of $G_{2}$ and there exists an object $e$ such that $e \in$ (the edges of $\left.G_{1}\right) \backslash$ (the edges of $\left.p(n)\right)$ and $p(n+1)$ is supergraph of $p(n)$ extended by $e$ between vertices $v$ and $w$ or supergraph of $p(n)$ extended by $e$ between vertices $w$ and $v \cdot \mathcal{P}[0]$. For every natural number $k$ such that $\mathcal{P}[k]$ holds $\mathcal{P}[k+1]$. For every natural number $k, \mathcal{P}[k]$.

(77) Let us consider a graph $G_{2}$, an object $v$, a finite set $V$, and a supergraph $G_{1}$ of $G_{2}$ extended by vertex $v$ and edges between $v$ and $V$ of $G_{2}$. Suppose $V \subseteq$ the vertices of $G_{2}$ and $v \notin$ the vertices of $G_{2}$. Then there exists a non empty, graph-yielding finite sequence $p$ such that

(i) $p(1)=G_{2}$, and

(ii) $p(\operatorname{len} p)=G_{1}$, and

(iii) len $p=\overline{\bar{V}}+2$, and

(iv) $p(2)$ is a supergraph of $G_{2}$ extended by $v$, and

(v) for every element $n$ of $\operatorname{dom} p$ such that $2 \leqslant n \leqslant \operatorname{len} p-1$ there exists a vertex $w$ of $G_{2}$ and there exists an object $e$ such that $e \in$ (the edges of $\left.G_{1}\right) \backslash($ the edges of $p(n))$ and $p(n+1)$ is supergraph of $p(n)$ extended by $e$ between vertices $v$ and $w$ or supergraph of $p(n)$ extended by $e$ between vertices $w$ and $v$.

The theorem is a consequence of (76).

(78) Let us consider a graph $G_{2}$, an object $v$, a non empty, finite set $V$, and a supergraph $G_{1}$ of $G_{2}$ extended by vertex $v$ and edges between $v$ and $V$ of $G_{2}$. Suppose $V \subseteq$ the vertices of $G_{2}$ and $v \notin$ the vertices of $G_{2}$. Then there exists a non empty, graph-yielding finite sequence $p$ such that

(i) $p(1)=G_{2}$, and

(ii) $p(\operatorname{len} p)=G_{1}$, and 
(iii) len $p=\overline{\bar{V}}+1$, and

(iv) there exists a vertex $w$ of $G_{2}$ and there exists an object $e$ such that $e \in$ (the edges of $\left.G_{1}\right) \backslash\left(\right.$ the edges of $G_{2}$ ) and $p(2)$ is supergraph of $G_{2}$ extended by $v, w$ and $e$ between them or supergraph of $G_{2}$ extended by $w, v$ and $e$ between them, and

(v) for every element $n$ of $\operatorname{dom} p$ such that $2 \leqslant n \leqslant \operatorname{len} p-1$ there exists a vertex $w$ of $G_{2}$ and there exists an object $e$ such that $e \in$ (the edges of $\left.G_{1}\right) \backslash$ (the edges of $\left.p(n)\right)$ and $p(n+1)$ is supergraph of $p(n)$ extended by $e$ between vertices $v$ and $w$ or supergraph of $p(n)$ extended by $e$ between vertices $w$ and $v$.

The theorem is a consequence of (76).

(79) Let us consider a finite, simple graph $G$, a set $W$, and a subgraph $H$ of $G$ induced by $W$. Then there exists a non empty, finite, simple, graphyielding finite sequence $p$ such that

(i) $p(1) \approx H$, and

(ii) $p(\operatorname{len} p)=G$, and

(iii) len $p=G$.order ()$-H$.order ()$+1$, and

(iv) for every element $n$ of $\operatorname{dom} p$ such that $n \leqslant \operatorname{len} p-1$ there exists an object $v$ and there exists a finite set $V$ such that $v \in$ (the vertices of $G) \backslash($ the vertices of $p(n))$ and $V \subseteq$ the vertices of $p(n)$ and $p(n+1)$ is a supergraph of $p(n)$ extended by vertex $v$ and edges between $v$ and $V$ of $p(n)$.

Proof: Define $\mathcal{P}$ [natural number] $\equiv$ for every finite, simple graph $G$ for every set $W$ for every subgraph $H$ of $G$ induced by $W$ such that $G$.order ()$-H$.order ()$=\$_{1}$ there exists a non empty, finite, simple, graph-yielding finite sequence $p$ such that $p(1) \approx H$ and $p(\operatorname{len} p)=G$ and len $p=G \cdot \operatorname{order}()-H \cdot \operatorname{order}()+1$ and for every element $n$ of $\operatorname{dom} p$ such that $n \leqslant \operatorname{len} p-1$ there exists an object $v$ and there exists a finite set $V$ such that $v \in($ the vertices of $G) \backslash($ the vertices of $p(n))$ and $V \subseteq$ the vertices of $p(n)$ and $p(n+1)$ is a supergraph of $p(n)$ extended by vertex $v$ and edges between $v$ and $V$ of $p(n)$.

$\mathcal{P}[0]$. For every natural number $k$ such that $\mathcal{P}[k]$ holds $\mathcal{P}[k+1]$. For every natural number $k, \mathcal{P}[k]$.

(80) Let us consider a finite, simple graph $G$. Then there exists a non empty, finite, simple, graph-yielding finite sequence $p$ such that

(i) $p(1)$ is trivial and edgeless, and

(ii) $p($ len $p)=G$, and 
(iii) $\operatorname{len} p=G$.order(), and

(iv) for every element $n$ of $\operatorname{dom} p \operatorname{such}$ that $n \leqslant \operatorname{len} p-1$ there exists an object $v$ and there exists a finite set $V$ such that $v \in$ (the vertices of $G) \backslash($ the vertices of $p(n))$ and $V \subseteq$ the vertices of $p(n)$ and $p(n+1)$ is a supergraph of $p(n)$ extended by vertex $v$ and edges between $v$ and $V$ of $p(n)$.

The theorem is a consequence of (79) and (52).

The scheme FinSimpleGraphs deals with a unary predicate $\mathcal{P}$ and states that

(Sch. 5) For every finite, simple graph $G, \mathcal{P}[G]$

provided

- for every trivial, edgeless graph $G, \mathcal{P}[G]$ and

- for every finite, simple graph $G_{2}$ and for every object $v$ and for every finite set $V$ and for every supergraph $G_{1}$ of $G_{2}$ extended by vertex $v$ and edges between $v$ and $V$ of $G_{2}$ such that $v \notin$ the vertices of $G_{2}$ and $V \subseteq$ the vertices of $G_{2}$ and $\mathcal{P}\left[G_{2}\right]$ holds $\mathcal{P}\left[G_{1}\right]$.

Now we state the propositions:

(81) Let us consider a non empty, graph-yielding finite sequence $p$. Suppose $p(1)$ is simple and for every element $n$ of $\operatorname{dom} p$ such that $n \leqslant \operatorname{len} p-1$ there exists an object $v$ and there exists a set $V$ such that $p(n+1)$ is a supergraph of $p(n)$ extended by vertex $v$ and edges between $v$ and $V$ of $p(n)$. Then $p(\operatorname{len} p)$ is simple.

Proof: Define $\mathcal{Q}$ [natural number] $\equiv$ if $\$_{1} \leqslant$ len $p$, then there exists an element $k$ of $\operatorname{dom} p$ such that $\$_{1}=k$ and $p(k)$ is simple. $\mathcal{Q}[1]$. For every non zero natural number $m$ such that $\mathcal{Q}[m]$ holds $\mathcal{Q}[m+1]$. For every non zero natural number $m, \mathcal{Q}[m]$.

(82) Let us consider a finite, simple, connected graph $G$. Then there exists a non empty, finite, simple, connected, graph-yielding finite sequence $p$ such that

(i) $p(1)$ is trivial and edgeless, and

(ii) $p(\operatorname{len} p)=G$, and

(iii) len $p=G$.order(), and

(iv) for every element $n$ of $\operatorname{dom} p$ such that $n \leqslant \operatorname{len} p-1$ there exists an object $v$ and there exists a non empty, finite set $V$ such that $v \in($ the vertices of $G) \backslash($ the vertices of $p(n))$ and $V \subseteq$ the vertices of $p(n)$ and $p(n+1)$ is a supergraph of $p(n)$ extended by vertex $v$ and edges between $v$ and $V$ of $p(n)$. 
Proof: Define $\mathcal{P}$ [natural number] $\equiv$ for every finite, simple, connected graph $G$ such that $G$.order ()$=\$_{1}$ there exists a non empty, finite, simple, connected, graph-yielding finite sequence $p$ such that $p(1)$ is trivial and edgeless and $p(\operatorname{len} p)=G$ and len $p=G$.order () and for every element $n$ of $\operatorname{dom} p$ such that $n \leqslant \operatorname{len} p-1$ there exists an object $v$ and there exists a non empty, finite set $V$ such that $v \in$ (the vertices of $G$ ) \(the vertices of $p(n))$ and $V \subseteq$ the vertices of $p(n)$ and $p(n+1)$ is a supergraph of $p(n)$ extended by vertex $v$ and edges between $v$ and $V$ of $p(n)$.

$\mathcal{P}[1]$. For every non zero natural number $k$ such that $\mathcal{P}[k]$ holds $\mathcal{P}[k+$ $1]$. For every non zero natural number $k, \mathcal{P}[k]$.

The scheme FinSimpleConnectedGraphs deals with a unary predicate $\mathcal{P}$ and states that

(Sch. 6) For every finite, simple, connected graph $G, \mathcal{P}[G]$

provided

- for every trivial, edgeless graph $G, \mathcal{P}[G]$ and

- for every finite, simple, connected graph $G_{2}$ and for every object $v$ and for every non empty, finite set $V$ and for every supergraph $G_{1}$ of $G_{2}$ extended by vertex $v$ and edges between $v$ and $V$ of $G_{2}$ such that $v \notin$ the vertices of $G_{2}$ and $V \subseteq$ the vertices of $G_{2}$ and $\mathcal{P}\left[G_{2}\right]$ holds $\mathcal{P}\left[G_{1}\right]$.

Now we state the proposition:

(83) Let us consider a non empty, graph-yielding finite sequence $p$. Suppose $p(1)$ is simple and connected and for every element $n$ of $\operatorname{dom} p$ such that $n \leqslant \operatorname{len} p-1$ there exists an object $v$ and there exists a non empty set $V$ such that $p(n+1)$ is a supergraph of $p(n)$ extended by vertex $v$ and edges between $v$ and $V$ of $p(n)$. Then $p(\operatorname{len} p)$ is simple and connected.

Proof: Define $\mathcal{Q}\left[\right.$ natural number] $\equiv$ if $\$_{1} \leqslant$ len $p$, then there exists an element $k$ of $\operatorname{dom} p$ such that $\$_{1}=k$ and $p(k)$ is simple and connected. $\mathcal{Q}[1]$.

For every non zero natural number $m$ such that $\mathcal{Q}[m]$ holds $\mathcal{Q}[m+1]$. For every non zero natural number $m, \mathcal{Q}[m]$.

\section{REFERENCES}

[1] Grzegorz Bancerek and Krzysztof Hryniewiecki. Segments of natural numbers and finite sequences. Formalized Mathematics, 1(1):107-114, 1990.

[2] Lowell W. Beineke and Robin J. Wilson, editors. Selected Topics in Graph Theory. Academic Press, London, 1978. ISBN 0-12-086250-6.

[3] John Adrian Bondy and U. S. R. Murty. Graph Theory. Graduate Texts in Mathematics, 244. Springer, New York, 2008. ISBN 978-1-84628-969-9.

[4] Adam Grabowski, Artur Korniłowicz, and Adam Naumowicz. Four decades of Mizar. Journal of Automated Reasoning, 55(3):191-198, 2015. doi:10.1007/s10817-015-9345-1 
[5] Sebastian Koch. About supergraphs. Part I. Formalized Mathematics, 26(2):101-124, 2018. doi $10.2478 /$ forma-2018-0009.

[6] Sebastian Koch. About supergraphs. Part II. Formalized Mathematics, 26(2):125-140, 2018. doi:10.2478/forma-2018-0010

[7] Gilbert Lee and Piotr Rudnicki. Alternative graph structures Formalized Mathematics, 13(2):235-252, 2005.

[8] Klaus Wagner. Graphentheorie. B.I-Hochschultaschenbücher; 248. Bibliograph. Inst., Mannheim, 1970. ISBN 3-411-00248-4.

[9] Robin James Wilson. Introduction to Graph Theory. Oliver \& Boyd, Edinburgh, 1972. ISBN 0-05-002534-1.

Accepted May 27, 2019 